\title{
Performance evaluation of a distributed application performance monitor
}

\author{
R. J. Friedrich ${ }^{a}$ and J. A. Rolia ${ }^{b}$ \\ ${ }^{a}$ Hewlett-Packard Laboratories, Hewlett-Packard Company, \\ Palo Alto, California, USA 94304; richf@hpl.hp.com \\ ${ }^{b}$ Department of Systems and Computer Engineering, Carleton University, \\ Ottawa, Canada KIS 5B6; jar@sce.carleton.ca
}

\begin{abstract}
The Distributed Measurement System (DMS) is a software-based measurement infrastructure for monitoring the performance of distributed application systems. In this paper we evaluate DMS in two configurations: a monitor for quality of service and a collector for model building parameters. Three distributed application workload types are defined and a model for DMS is given. The model parameters for DMS are based on measurement data from an implementation of DMS for the Open Software Foundation's Distributed Computing Environment. We use the model with our workloads to consider the impact of DMS on processor and network utilization and on workload responsiveness. We show how the various factors that control DMS affect its overhead. Lastly, the scalability of DMS is considered for large distributed environments. Our results indicate that DMS is well suited for monitoring QoS and supporting workload characterization for model building.
\end{abstract}

\section{Keywords}

Performance models, distributed applications, client-server, performance monitors

\section{INTRODUCTION}

Collecting, analyzing and modeling application workload data is necessary for predicting and managing the dynamic behavior of distributed applications. Researchers at Hewlett-Packard developed the Distributed Measurement System (DMS) [6], a software-based distributed application performance monitor, to address these measurement needs. Using the terminology of the Reference Model for Open Distributed Processing (RM-ODP) [1], DMS provides correlated performance metrics across objects (application components) and their channels (network communication), integrates disparate performance measurement interfaces from a node's nucleus object (operating system), and efficiently transports collected data from network nodes to management stations. The data collected by DMS is useful to application designers, model developers, quality of service (QoS) monitors and distributed application managers.

DMS sensors (instrumentation) are designed and placed to facilitate data collection for application workload characterization. This data is used to build and validate predictive performance models of distributed applications. For example, the data can be used to capture the parameters for Layered Queueing Models (LQM) [3][11] of distributed applications. These models are similar to Queueing Network Models [9], which are used to estimate a workload's contention for processors and input-output subsystems, but also capture software interactions between capsules (operating system processes) that can affect application throughput and 
responsiveness. Quantitative performance models provide the basis for evaluating the partitioning of application functionality, upgrading the performance and scalability of existing applications, developing new application designs, and planning capacity. DMS is itself a distributed application and we use it in this paper to provide an experimental application to validate these performance modeling methods.

Several requirements must be satisfied to support efficient QoS monitoring of applications in open distributed processing environments. First, standard metrics must be defined so that metrics collected from heterogeneous sources are comparable. Second, pervasive instrumentation must be implemented to support the collection of the standard metrics. Third, standard access and control interfaces must be defined that permit the efficient collection and transportation of performance data from the collection source to the analysis destination. Finally, monitoring QoS in operational environments implies strict limits on collection overhead to minimize monitoring perturbation. Strict utilization goals were established for QoS monitoring: less than 5\% CPU utilization of a network node to collect data and less than 5\% network utilization to transport data.

In this paper we describe a performance model that characterizes the resource consumption of key DMS components. Three distributed application workload types are defined and used in the evaluation. We then use analytic modeling techniques to predict the impact of DMS on resource utilization and application responsiveness for the workloads. A utilization analysis estimates the change in processor and network utilization due to DMS. A LQM is used to predict the impact of DMS on the responsiveness of the workloads. We also analyze DMS scalability for very large distributed environments.

\section{DISTRIBUTED MEASUREMENT SYSTEM - DMS}

DMS collects and reports on data for the performance management of distributed application systems. DMS defines a common set of performance metrics and instrumentation to ensure consistent collection and reporting across heterogeneous nodes. It also creates standard application programming interfaces to DMS objects to ensure pervasive measurement support for performance metrics. The DMS objects shown in Figure 1 are capable of monitoring applications developed using distributed technologies such as DCE and CORBA. This research has lead to the definition of an instrumentation specification for the OSF DCE [5] targeted for implementation in OSF DCE release 1.2. DMS also provides an infrastructure for integrating performance measurement interfaces from sources such as the host operating system into a single unified measurement and reporting infrastructure. This results in a seamless, integrated view of the behavior of a distributed application in a heterogeneous environment.

\subsection{DMS architecture}

The DMS architecture is briefly described here and illustrated in Figure 1. A more detailed discussion, including comparison to other monitors such as JEWEL [8] and ZM4 [2], can be found in [6].

Sensor objects are instances of performance metrics that accumulate distributional statistics. Sensors support varying information levels that specify the amount and detail of data collected. For example, higher moments that describe the variance, skew, and n-percentile of a distribution can be collected. Sensors also provide user definable thresholds and only report data when a threshold condition is true. Sensors collect data on a per object member function granularity for all capsule interface methods and report it on a user configured frequency.

Since objects might be executed by different threads, sensors are reentrant. A temporary sensor instance is allocated for each capsule thread (if the sensor is active) and must be combined with the global sensor object at the end of the member function execution. Updates of the global sensor object are protected with concurrency control mechanisms. 
Figure 1 The DMS architecture. DMS objects are represented as oval figures and interfaces are shown as rectangles. The control and data paths are shown by arrows.

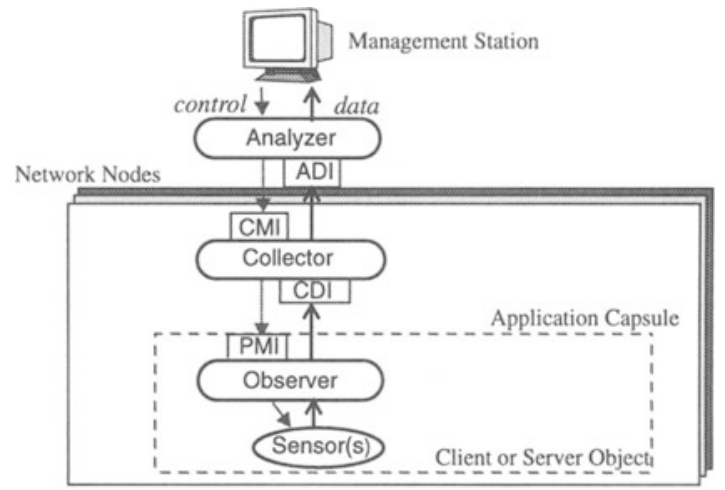

An Observer object within each instrumented capsule implements a sensor access and control interface named the Performance Measurement Interface (PMI). It minimizes in-line overhead by allowing the sensors to defer some computation and off-loads sensors of the need to manage and transmit data. The observer transmits intervalized sensor data from the local capsule's address space into the collector object. Multiple nonzero sensor values are transferred at the same time to minimize IPC cost.

The Collector is a node level object that controls sensors and performs node-level sensor data management. It provides transparent network access and control of sensors for higher levels of the DMS architecture using the Collector Measurement Interface (CMI). The collectors obtain sensor data from all observers on the node using the Collector Data Interface (CDI). The observer periodically "pushes" sensor data to the collector using the CDI. The CDI eliminates the need for polling of sensors and provides an asynchronous data transport channel for acquiring sensor data.

An Analyzer object analyzes the data gathered by collector objects. It computes the higher moments of the collected data, correlates data from application elements residing on different nodes, and prepares data for expert system or human analysis. The collector periodically pushes sensor data collected from the observers on a node to the analyzer via the Analyzer Data Interface (ADI). The ADI is required to support a remote procedure call (RPC) interface since it most likely resides on a node elsewhere in the network.

We have used optimization techniques to minimize the amount of CPU and network utilization required and to improve scalability. Specifically, the DMS architecture uses:

- sensors that periodically report summarized data,

- thresholds so that only exceptions are reported in a normal executing environment,

- and non-polling, bulk transfer algorithms for network requests to minimize bandwidth use.

Other performance monitors focus on distributed debugging with either expensive custom hardware monitors or highly intrusive software-based event tracing [12]. Although distributed debugging is a very important feature in distributed systems management these approaches have too high an overhead for the continuous monitoring of distributed applications in operational environments.

\subsection{DMS performance model}

A prototype of the DMS architecture was implemented on DCE. It supports the sensor, observer, collector, and analyzer objects. The use of DCE as the prototype's distribution infrastructure impacts only the placement of sensors and the implementation of observers. All other DMS objects are independent of the distribution infrastructure. 
Sensors were developed and placed within the DCE runtime library (RTL). These sensors are available to all DCE clients and servers without modifying application source code. The observer is implemented as a special timer thread within the RTL. The collector was implemented as a daemon process and it communicated with all the observers on the node via IPC using the PMI and CDI interfaces. The analyzer was implemented as a daemon and it communicated with all the collectors in the network via RPC.

Figure 2 Application capsule with DMS objects. Labels correspond to functions or events that are modeled as described in the text and in Table 1.

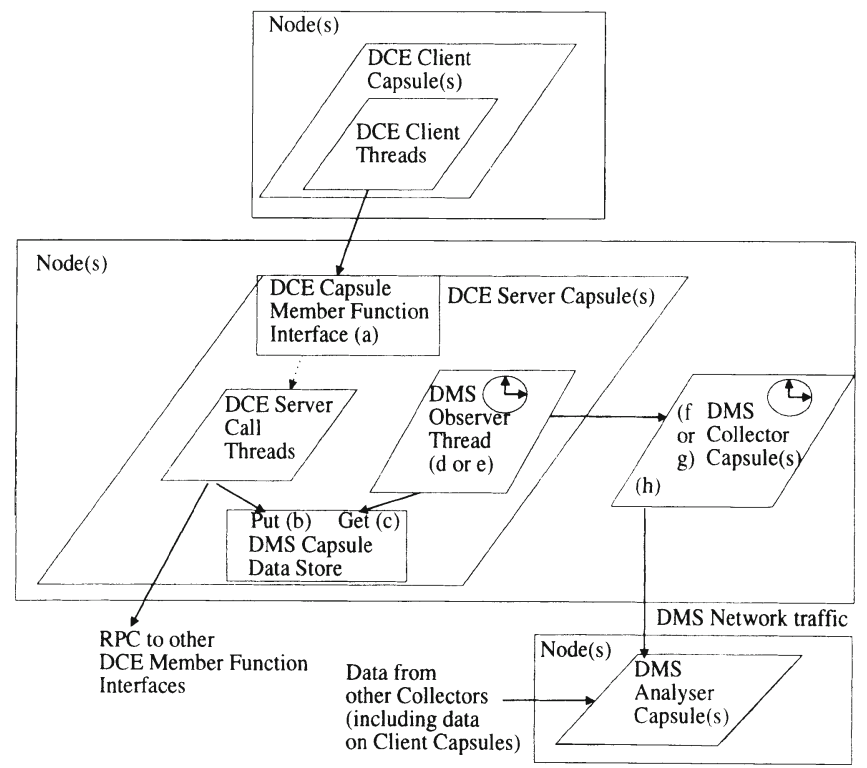

Figure 2 illustrates a distributed application along with DMS. In the figure parallelograms represent capsules and their internal threads. Arrows represent synchronous requests for service, a dotted arrow indicates that a request for service is mapped to the next available application call thread. The RTL within an application capsule includes the pool of application call threads and a single observer thread. A capsule global data store is used to maintain the sensor data for individual member functions for the capsule. It is protected by mutual exclusion and is a potential source of contention for the capsule's threads. The observer thread and collector capsule are driven by timers that control their wake-up frequency. The labels $(\mathrm{a}, \mathrm{b}, \mathrm{c}, \ldots, \mathrm{h})$ indicate where monitoring overhead is incurred. Example values for the overheads and the descriptions of the labels are given in Table 1. We assume that monitoring does not increase disk queueing times; therefore, disk residence times are assumed to be constant.

Our performance model characterizes the four functions of DMS that contribute most to its utilization of system resources. These are:

1. the cost of sensor instrumentation during execution of a member function;

2. the update of a capsule's global data store (DS) at the end of an RPC request;

3. the gathering of data by an observer and its push of data to the collector;

4. and the gathering of data by the collector and its push of data to an analyzer.

The pathlengths for these functions were measured directly or estimated based on previously measured pathlengths for key services such as mutex operations and RPCs. In general, measuring increasingly detailed sensor information requires more instructions to collect, produces more data, and requires more instructions and network bandwidth for communication. 
The communication mechanism used to exchange data between observers and the collector, RPC versus shared memory IPC, also has an impact on DMS overhead.

Table 1: DMS functions and costs on an HP9000/720 running HP-UX 9.02

\begin{tabular}{lcr}
\hline Function/event & $\begin{array}{c}\text { Figure 2 } \\
\text { label }\end{array}$ & $\begin{array}{r}\text { Cost } \\
\text { (instructions) }\end{array}$ \\
\hline Total sensor collection & $\mathrm{a}$ & 280 \\
Update global sensor data store (DS) & $\mathrm{b}$ & 232 \\
Observer get sensor data from DS & $\mathrm{c}$ & 232 \\
Observer report data (via RPC) & $\mathrm{d}$ & 21,264 \\
Observer report data (via shared memory) & $\mathrm{e}$ & 2,264 \\
Collector receive data (via RPC) & $\mathrm{f}$ & 430,280 \\
Collector receive data (via shared memory) & $\mathrm{g}$ & 46,280 \\
Collector reports data (via RPC) & $\mathrm{h}$ & 51,200 \\
\hline
\end{tabular}

Our experimental design considers four factors:

1. strategies for applying DMS (which determines the number of active sensors per object member, their information level, and the frequency of reporting data);

2. the number of object member functions monitored per server capsule;

3. the communication mechanism between observer and collector;

4. and the workload type.

We now discuss the levels of the first three factors and their corresponding resource costs. The workload types are discussed in section 3 .

First we assume that there arc no active sensors. This gives us our baseline performance without monitoring. In the second case we examine the cost of monitoring application QoS and in the third case we examine the cost of collecting the data necessary for workload characterization and model building. These cases are considered for both RPC and shared memory communication between observer and collector.

In the QoS monitoring case, 2 sensors are active for each application capsule object member function. These two sensors provide data for response time and throughput. Our motivation for collecting these values is from QoS requirements for transaction processing systems. Such requirements are often expressed in the form "under a load of 30 transactions per second, $90 \%$ of the transactions must complete in less than 5 seconds." The response time (timer) sensor collects percentile data using the $\mathrm{P}^{2}$ algorithm [7] and the throughput (counter) sensor collects counts, sums and sum of squares (used by the analyzer to compute means and standard deviations.) In this example we define the information level of the response time sensor as the percentile information level and the throughput sensor as the basic information level.

In the model building case we consider detailed measurements with 5 sensors active for all capsule member functions. These 5 sensors provide data for response time, throughput, network bytes transmitted and received, and queueing time (for server objects that do not have an available thread to handle an incoming service request). All 5 of these sensors are set to the basic information level. In addition, once per reporting interval, the observer collects nucleus (operating system) metrics for capsule (process) CPU resource consumption and disk $\mathrm{I} / \mathrm{O}$ counts using an OS sensor. The sensor cost and data size values are summarized in Table 2.

Note that when DMS is used for continuous application QoS monitoring thresholds are likely to be employed to reduce the amount of data collected and analyzed. Sensors with thresholds report data only when a user configured requirement is exceeded; for example, when $10 \%$ of the client response times exceed 5 seconds. The use of thresholds significantly reduces the amount of data transmitted between observer and collector, and collector and analyzer. This limits the collection and communication overhead to only those member functions behaving in an abnormal manner. At first we present results which assume that reporting always takes place. This gives us the worst case behavior for DMS. Later we describe the sensitivity of resource 
utilization to threshold values that result in a certain percentage of sensors reporting data.

Table 2: Sensor data size and cost on an HP9000/720 running HP-UX 9.02

\begin{tabular}{lrr}
\hline Sensor Type & $\begin{array}{r}\text { Data size } \\
\text { (bytes) }\end{array}$ & $\begin{array}{r}\text { Cost } \\
\text { (instructions) }\end{array}$ \\
\hline Timer sensor (percentile information level) & 64 & 100 \\
Counter sensor (basic information level) & 24 & 40 \\
OS sensor (basic information level) & 24 & 500 \\
\hline
\end{tabular}

The number of member functions used in the model is the member functions per capsule that were invoked at least once during the reporting interval (and since not all member functions are invoked every reporting interval, this parameter represents the most frequently accessed functions for an object that may export many more member functions than this value). The number of member functions and the number of active sensors per member function determines the sensor CPU consumption and the amount of data that is generated. We use the model to find the number of member functions that can be monitored without exceeding the 5\% CPU utilization limit.

Each time a server object member function is executed at the start of an RPC request the function's thread creates a thread-local copy of data for the member's active sensors. At the completion of the RPC request the local data is used to cumulatively update the capsule's global sensor data store. A lock is acquired prior to updating the data store. On average 24 bytes of data is written to the data store for the basic information level and 40 additional bytes are written for the percentile information level.

Periodically the observer reports sensor data to the collector. Two IPC mechanisms are considered. The first utilizes RPC and the second uses shared memory with locks. In DMS the observer reporting period can be controlled. In our performance study it is set at either 5, 30 or 150 seconds.

The collector also incurs additional CPU resource consumption when it reports sensor data to the analyzer using an RPC. The volume of the data is calculated and used to estimate the network utilization due to monitoring. In our study the collector reports its data for the node to the analyzer at one-half the frequency of the observer, i.e. every 10,60 or 300 seconds.

The CPU instructions and number of bytes generated for our factor combinations were determined and used to parameterize the model. Example values for the QoS monitoring of the Light workload with one member function per capsule object is shown in Table 1. Values were determined for the cases with 1,25, and 50 object member functions per capsule. The collector instructions and number of bytes depends on the number of instrumented capsules. We assume there are ten capsules per node for the numbers given in Table 1.

\section{DISTRIBUTED APPLICATION WORKLOADS}

We have defined three workloads to evaluate the performance characteristics of DMS. These workloads are named light, medium, and heavy based on their relative CPU and input-output subsystem resource utilization. Specific workload characteristics are summarized in Table 3.

Table 3: Workload Characteristics

\begin{tabular}{|c|c|c|c|c|c|c|}
\hline $\begin{array}{l}\text { Application } \\
\text { workload }\end{array}$ & $\begin{array}{r}\text { Instructions } \\
\text { per } \\
\text { transaction }\end{array}$ & $\begin{array}{r}\text { Clients } \\
\text { per } \\
\text { server } \\
(N)\end{array}$ & $\begin{array}{r}\text { Think } \\
\text { time } \\
(\text { Z sec })\end{array}$ & $\begin{array}{r}\text { Visits to } \\
\text { server } \\
(V)\end{array}$ & $\begin{array}{r}\text { Number of } \\
\text { servers per } \\
\text { node }\end{array}$ & $\begin{array}{c}\text { MIP Rate } \\
\text { (that results } \\
\text { in } 60 \% \text { CPU } \\
\text { utilization) }\end{array}$ \\
\hline Light & 75,000 & 100 & 10 & 1 & 10 & $12,500,000$ \\
\hline Medium & 300,000 & 100 & 30 & 3 & 10 & $50,000,000$ \\
\hline Heavy & $3,000,000$ & 10 & 180 & 30 & 10 & $83,333,333$ \\
\hline
\end{tabular}


In actuality these workloads are based on experiences with commercial applications. The light workload reflects an on-line transaction processing application consisting of simple queries of medium size (100 MB) databases with minimal CPU and disk resource demand but with a frequent issue rate (similar to TPC-A). An example of this workload is a banking application that provides customers with account information and financial transactions such as account debits and credits. Typically a large number of clients access a given server, 100 in this case.

The medium workload is similar to the light but with increased query complexity. Consequently, the CPU and disk resource utilization is larger than in the light case and the user think time is larger. An example of this class of applications is an inventory control program for a manufacturing company where parts are entered and deleted from inventory based on incoming customer orders and outgoing shipments. The number of clients per server is still 100 .

The heavy workload is based on a decision support application where a user issues complex queries against a large (greater than $1 \mathrm{~GB}$ ) database. The CPU and disk resource utilization is much larger than for the previous two workloads. This workload has a much smaller number of clients per server due to the client's workload intensity. This workload also has a much larger think time reflecting user analysis of the results. Examples of this workload include retail companies that use data warehouses containing historical data for trend analysis.

In our study the applications all have the same software capsule architecture as illustrated in Figure 2. Clients reside on dedicated nodes and make use of a server node. The server node has ten managed capsules (server processes) and a collector. The processing power (MIP rates) of the server nodes has been chosen so that the processor utilization is $60 \%$ for the baseline case without monitoring.

\section{DMS PERFORMANCE EVALUATION}

We now evaluate the performance impact of DMS when monitoring the three workload types. We create baseline models for the three workloads that have no overhead due to DMS. A second set of models reflects the use of DMS to monitor application QoS. The third set estimates DMS performance to capture data needed to build and validate predictive distributed application performance models.

In the models the number of member functions is essentially the number of different services a server provides. It also controls the amount of monitoring data that is captured. The number is set as either 1,25 or 50 . The second and third levels seem sufficiently large to characterize real applications and were chosen to stress DMS.

The utilization law $\mathrm{U}=\mathrm{X} \mathrm{D}$ is used to compute DMS processor and network utilization. The processor utilization is the sum of the utilizations due to collector, observer, and sensor overheads. Models for the demands of these three components were discussed in section 2.2. The throughputs of the collector and observer instrumentation are based on their reporting frequency. The throughputs of the sensor instrumentation are determined by the number of clients $\mathrm{N}$, their number of visits $\mathrm{V}$ to the server per think period, and their think times $\mathrm{Z}$, related by the formula $X=N V / Z$. The values for the workload's $N, V$, and $Z$ are given in Table 3 .

Our model is a closed model so the actual throughput is $X=N /(Z+R)$ where $R$ is the (unknown) response time of a customer, so the sensor throughput and overhead estimates are high. However, as we shall show, the sensors contribute very little to the overall overhead of DMS so this pessimistic approximation does not significantly affect the results.

Network utilization depends on the number of different sensors that report data, the amount of data reported for each sensor, and the reporting frequencies of the observers. Network utilization by the workload itself is not modeled.

We are also interested in understanding how DMS affects client response times. To study this we use the method of layers (MOL) [11]. It is a mean value analysis technique that takes into account software interactions in distributed application systems. 
Figure 3 DMS CPU utilization estimates for the QoS monitoring case $\mathrm{QOS}_{\mathrm{A}}$ for the three workloads. The observer-collector IPC method is plotted for RPC (back row) and Shared Memory (front row). Utilization is plotted for 1, 25, and 50 object member functions per capsule for each workload.

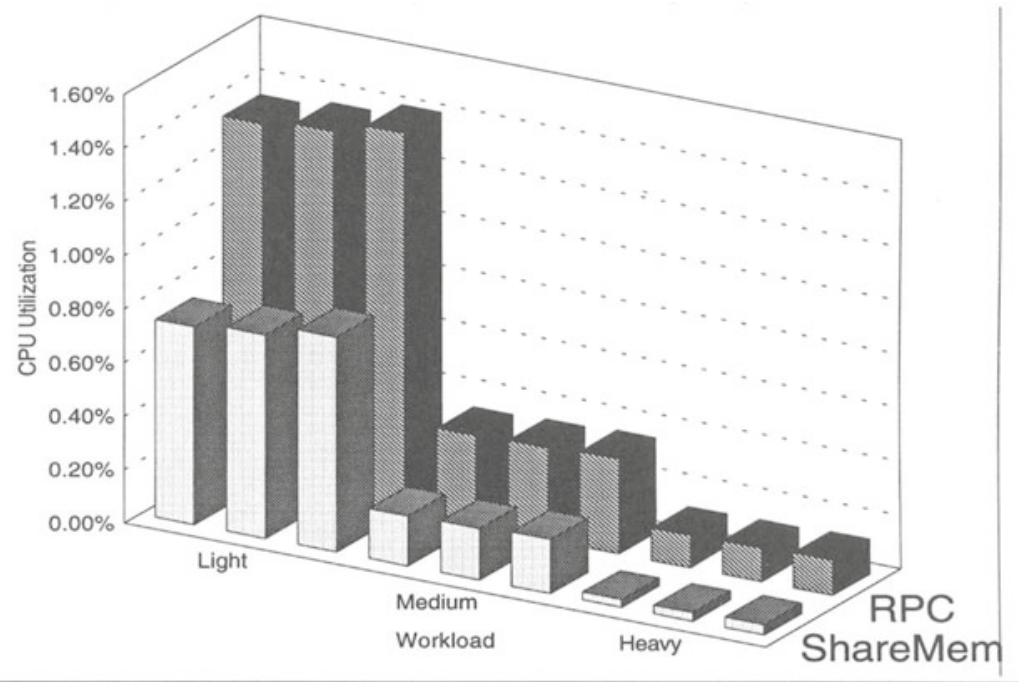

Figure 4 DMS CPU utilization estimates for the QoS monitoring cases as described in Table 4 for the Light workload only. The observer-collector IPC method is RPC. Utilization is plotted for 1, 25, and 50 object member functions per capsule.

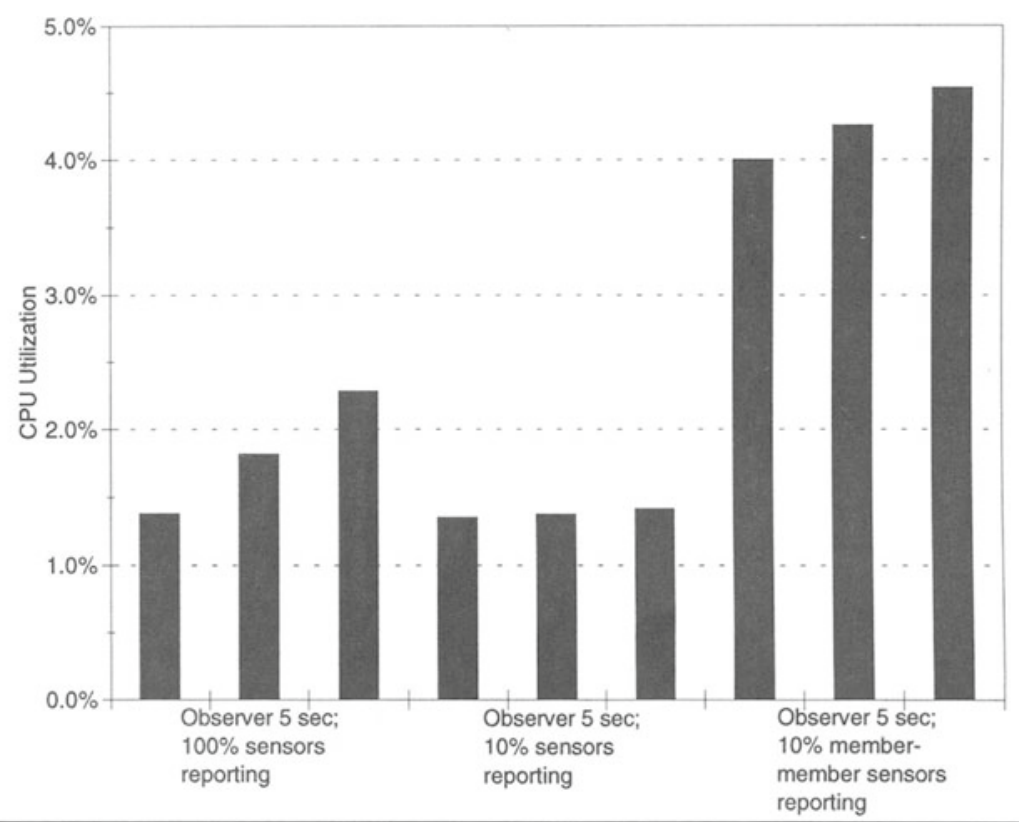




\subsection{CPU utilization}

\section{QoS monitoring impact on CPU utilization}

We consider three scenarios for monitoring quality of service labeled $\mathrm{QoS}_{\mathrm{A}}, \mathrm{QoS}_{\mathrm{B}}$ and $\mathrm{QoS}_{\mathrm{C}}$ and summarized in Table 4. In the $\mathrm{QoS}_{\mathrm{A}}$ and $\mathrm{QoS}_{\mathrm{B}}$ cases capsule object member function response times and throughputs are measured by DMS. Response times are recorded at the percentile information level and throughputs at the basic information level. In the $\mathrm{QoS}_{\mathrm{A}}$ case all of the sensor data is reported by the observers. In the $\mathrm{QoS}_{\mathrm{B}}$ and $\mathrm{QoS}_{\mathrm{C}}$ cases all of the data is collected but threshold values are set such that only $10 \%$ of the data is reported by an observer. Note that we consider $10 \%$ reporting a high (pessimistic) value.

Table 4: Model Factors for QoS Scenarios

\begin{tabular}{lrrrrrr}
\hline & $\begin{array}{r}\text { Number of } \\
\text { sensors per } \\
\text { member } \\
\text { function }\end{array}$ & $\begin{array}{r}\text { Additional } \\
\text { member- } \\
\text { member } \\
\text { sensors }\end{array}$ & $\begin{array}{r}\text { Sensors } \\
\text { above } \\
\text { thresh- } \\
\text { old }(\%)\end{array}$ & $\begin{array}{r}\text { Capsule } \\
\text { object } \\
\text { member } \\
\text { functions }\end{array}$ & $\begin{array}{c}\text { Observer- } \\
\text { Collector IPC } \\
\text { method }\end{array}$ & $\begin{array}{r}\text { Observer } \\
\text { reporting } \\
\text { period } \\
\text { (sec) }\end{array}$ \\
\hline $\mathrm{QoS}_{\mathrm{A}}$ & 2 & 0 & $100 \%$ & $1,25,50$ & RPC, Shared Memory & 5 \\
$\mathrm{QoS}_{\mathrm{B}}$ & 2 & 0 & $10 \%$ & $1,25,50$ & RPC, Shared Memory & 5 \\
$\mathrm{QoS}_{\mathrm{C}}$ & 2 & 10 & $10 \%$ & $1,25,50$ & RPC, Shared Memory & 5 \\
\hline
\end{tabular}

Table 5: Model Factors for Model Building Scenarios

\begin{tabular}{lrrrrrr}
\hline $\begin{array}{l}\text { Model } \\
\text { building } \\
\text { scenario }\end{array}$ & $\begin{array}{r}\text { Number of } \\
\text { sensors per } \\
\text { member } \\
\text { function }\end{array}$ & $\begin{array}{r}\text { Additional } \\
\text { member- } \\
\text { member } \\
\text { sensors }\end{array}$ & $\begin{array}{r}\text { Sensors } \\
\text { above } \\
\text { thresh- } \\
\text { old }(\%)\end{array}$ & $\begin{array}{r}\text { Capsule } \\
\text { object } \\
\text { member } \\
\text { functions }\end{array}$ & $\begin{array}{c}\text { Observer- } \\
\text { Collector IPC } \\
\text { method }\end{array}$ & $\begin{array}{r}\text { Observer } \\
\text { reporting } \\
\text { period } \\
\text { (sec) }\end{array}$ \\
\hline MdlBld $_{\mathrm{D}}$ & $5+2 \mathrm{OS}$ & 0 & $100 \%$ & $1,25,50$ & RPC, Shared Memory & 30 \\
MdlBld $_{\mathrm{E}}$ & $5+2 \mathrm{OS}$ & 10 & $100 \%$ & $1,25,50$ & RPC, Shared Memory & 30 \\
MdlBld $_{\mathrm{F}}$ & $5+2 \mathrm{OS}$ & 10 & $100 \%$ & $1,25,50$ & RPC, Shared Memory & 150 \\
\hline
\end{tabular}

The $\mathrm{QoS}_{\mathrm{C}}$ case considers a complex QoS management scenario. We assume that server member functions require service from other service providers (for example a security server or database system). In this case a member function's QoS depends on the QoS provided by its nested service providers. In this scenario's model each member function maintains timer sensors to record percentile level information for ten of its nested service providers. We label these member-member sensors.

Table 4 gives a summary of the factor levels for our three scenarios. Figure 3 shows the impact on CPU utilization for the three workload types. Results for the two alternatives for observer-collector communication, RPC and shared memory, are given. From the figure we see that DMS has its largest CPU impact on the light workload. Our name for the workload was chosen to suggest that a small number of instructions are required for each client visit to the server. However the Light client service demand of the server is small, so the relative sensor overhead is highest. Note that the overhead for the medium and heavy workloads is less than $0.5 \%$. Note that the use of shared memory for communication decreases processor utilization between $0.1 \%$ and $0.6 \%$ depending on the case. Figure 4 illustrates the CPU utilization of the various QoS monitoring configurations in Table 4 for the Light workload only.

Model building measurement impact on CPU utilization

Model building captures the resource demands needed to build predictive performance models of distributed applications [4] [10]. We consider three scenarios for model building labeled MdlBld ${ }_{D}$, MdlBld $_{E}$ and MdlBld $F$ and summarized in Table 5. The MdlBld $D$ and MdlBld $_{E}$ cases have 30 second observer periods while the MdlBld $F$ case has 150 second. The MdlBld $_{\mathrm{E}}$ and MdlBld $\mathrm{F}$ cases have additional instrumentation so that each member monitors its interactions with 10 of its nested service providers using member-member sensors set at the basic information level. 


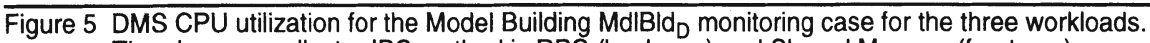
The observer-collector IPC method is RPC (back row) and Shared Memory (front row).

Utilization is plotted for 1, 25, and 50 object member functions per capsule for each workload.
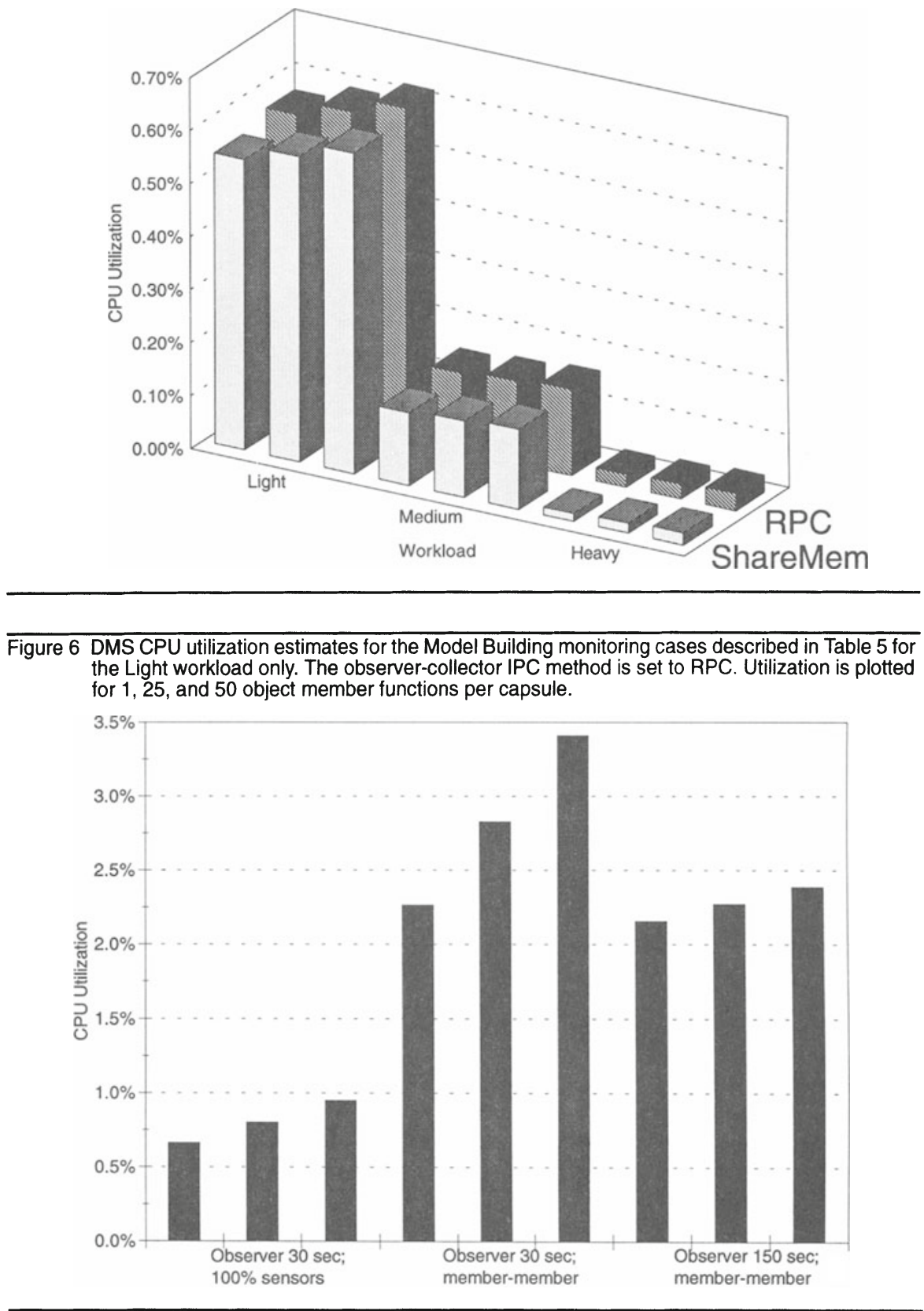
Figure 5 graphs the impact of DMS on CPU utilization for the three workload types. Results for the two alternatives for observer-collector communication, RPC and shared memory, are given. DMS has its largest CPU impact on the light workload but the impact is less than $0.60 \%$. Note that the use of shared memory for communication decreases utilization less than $0.10 \%$. Figure 6 graphs the impact on utilization for the Light workload for the RPC case for the three different model building configurations described in Table 5 .

\subsection{Network utilization}

Figure 7 illustrates the network utilization per collector for the three QoS configuration cases and the three Model Building cases. As expected QoS monitoring without effective threshold values (100\% of sensors reporting) has the highest network utilization while the more realistic $\mathrm{QoS}_{\mathrm{B}}$ case with $10 \%$ reporting is 10 times better -- only $0.05 \%$ utilization for 1000 sensors. For the model building cases, the MdlBld $\mathrm{E}$ case of 30 second observer period and member-member measurement has the highest network utilization as a function of number of sensors. The more realistic $\mathrm{MdlBld}_{\mathrm{F}}$ case of 150 second observer period and member-member measurement has the lowest network utilization of all the QoS and model building configurations -- only $0.12 \%$ for 2500 sensors.

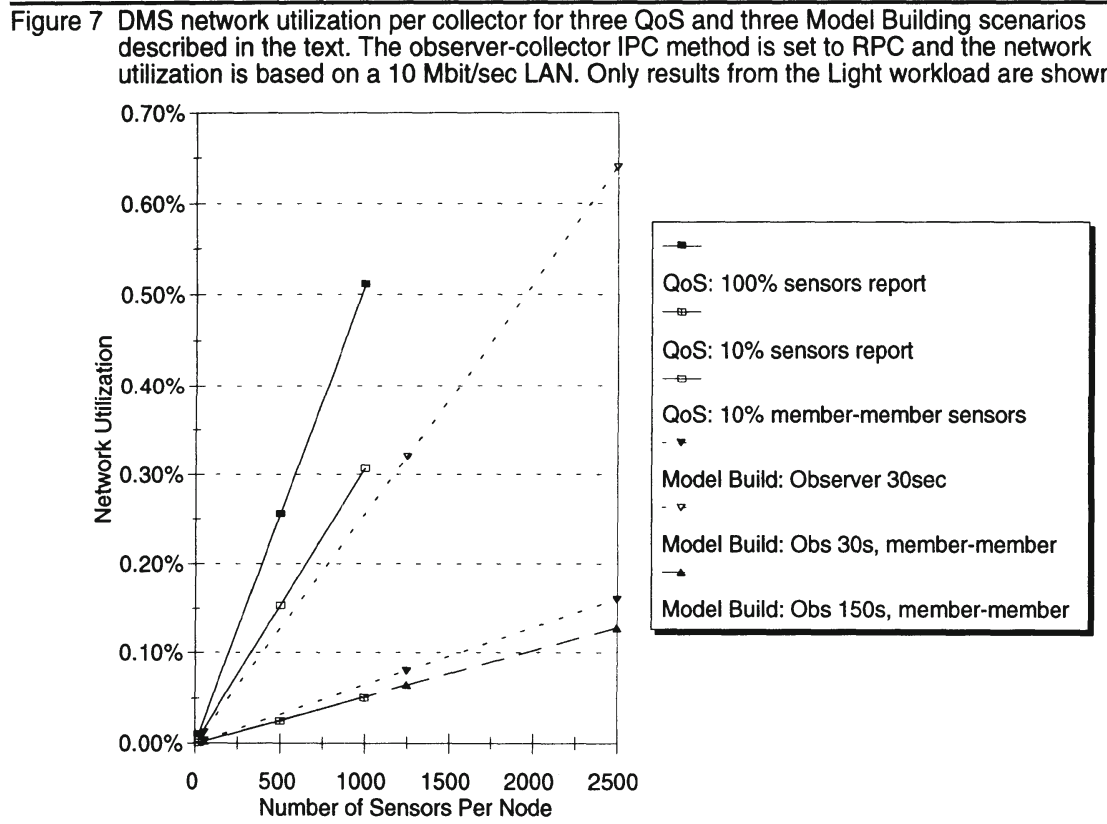

\subsection{Client response times}

A Layered Queueing Model (LQM) is created to study the impact of DMS overhead on client responsiveness for the light workload. The Method of Layers is used to estimate client response times. The accuracy of this analytic technique with respect to simulation is favorably demonstrated in [11].

There are several ways in which DMS contributes to response time delays. Greater contention for the processor will have an impact as will software contention for the capsule global data store. The method of layers takes both processor and software contention into account when estimating the response times. The impact of network overhead on client 
responsiveness is assumed to be low and constant and is not considered.

Seven cases were studied for the light workload: a baseline case with no monitoring overhead and the six cases identified in Table 4 and Table 5. In each case the number of server call threads was set to 10 and the number of member functions was 25 . Because of the high number of call threads there was effectively no software contention for the server capsules. Essentially a call thread was available whenever a call arrived. Figure 8 gives a client's mean residence time at its server capsule for these seven cases. The residence time includes the time a client spends completing the server member function (including processor and disk time), time executing the sensor instrumentation, and a visit to the capsule's global sensor data store.

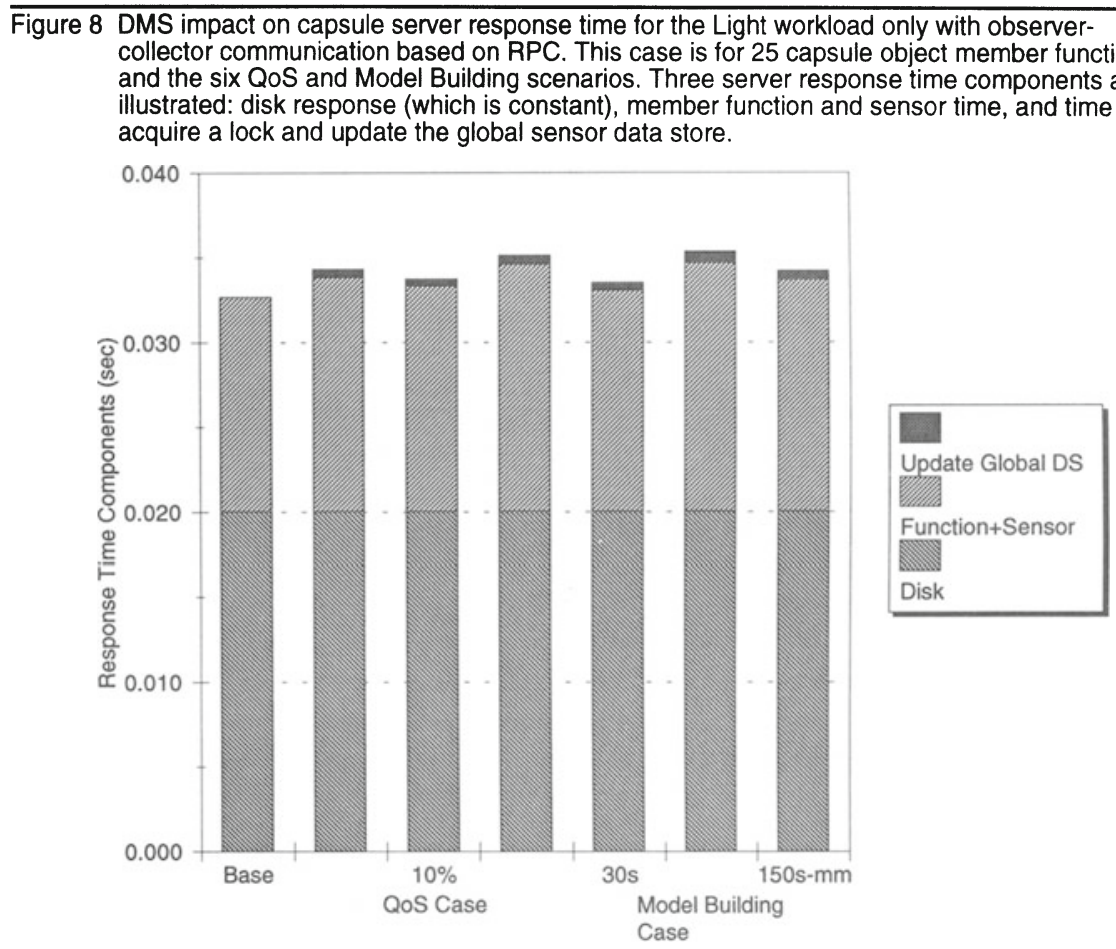

From analysis of detailed results not presented in this paper there is little contention for the data store lock. The change in residence time was all due to increased processor demand and competition for the processor. From Figure 8 we see that in the worst case the residence time increases by up to $8 \%$. This occurs in the MdlBld $\mathrm{E}$ model building case with members monitoring 10 nested service providers and an observer period of 30 seconds. When the observer period is increased to a more reasonable period of 150 seconds in the MdlBld $_{F}$ case the impact drops to $4.6 \%$. It is interesting to note that visiting the data store has a $1.5 \%$ impact on the residence time. This is the case even though the processor demand needed to access the store is small. When acquiring the store's mutex the thread must yield to other threads in the capsule. Thread scheduling for processor access is first come first served within the capsule, so if delayed, the thread must wait until threads with much larger service demands complete first.

The $\mathrm{QoS}_{\mathrm{B}}$ case with a 5 second observer period has a $3.2 \%$ impact on client residence times while the $\mathrm{QoS}_{\mathrm{C}}$ case has increases to $7.4 \%$. This can be reduced by increasing the observation period: 5 seconds is the minimum value possible under DMS, a more reasonable value may be 300 seconds. 
However, in all cases there was a less than $1 \%$ increase in response times viewed by end users at the client (note that Figure 8 graphs only the server response components). This response time includes the 0.3 seconds of processing time on a client's own node.

\subsection{Scalability}

Monitoring performance in large, heterogeneous environments requires a scalable measurement infrastructure. Several techniques were utilized in the design of the DMS to improve its scalability. Figure 9 predicts the scalability of DMS for QoS monitoring cases for a range of sensor reporting percentages of $1 \%, 10 \%$ and $100 \%$, and for observer periods of 5 and 150 seconds. For good application performance we have constrained the amount of DMS network utilization to $5 \%$ of a $10 \mathrm{Mbit} / \mathrm{sec} \mathrm{LAN}$. We have assumed that the application network requirements are met by this LAN technology and do not consider them further.

Scalability in this figure is the number of distinct nodes supporting a given number of sensors. The left-most bar indicates that DMS can support 1.46 million nodes where each node has 20 active sensors with a threshold level set such that no more than $1 \%$ of them report per 150 second observer period. The next bar indicates that 14.6 thousand nodes can be supported where each node has 20 active sensors with a threshold level set such that no more than $1 \%$ of them report per 5 second observer period. The trade-off for scalability is the number of nodes supported versus the latency in receiving sensor data. As expected the number of nodes that can be supported is inversely proportional to the number of sensors reporting data.

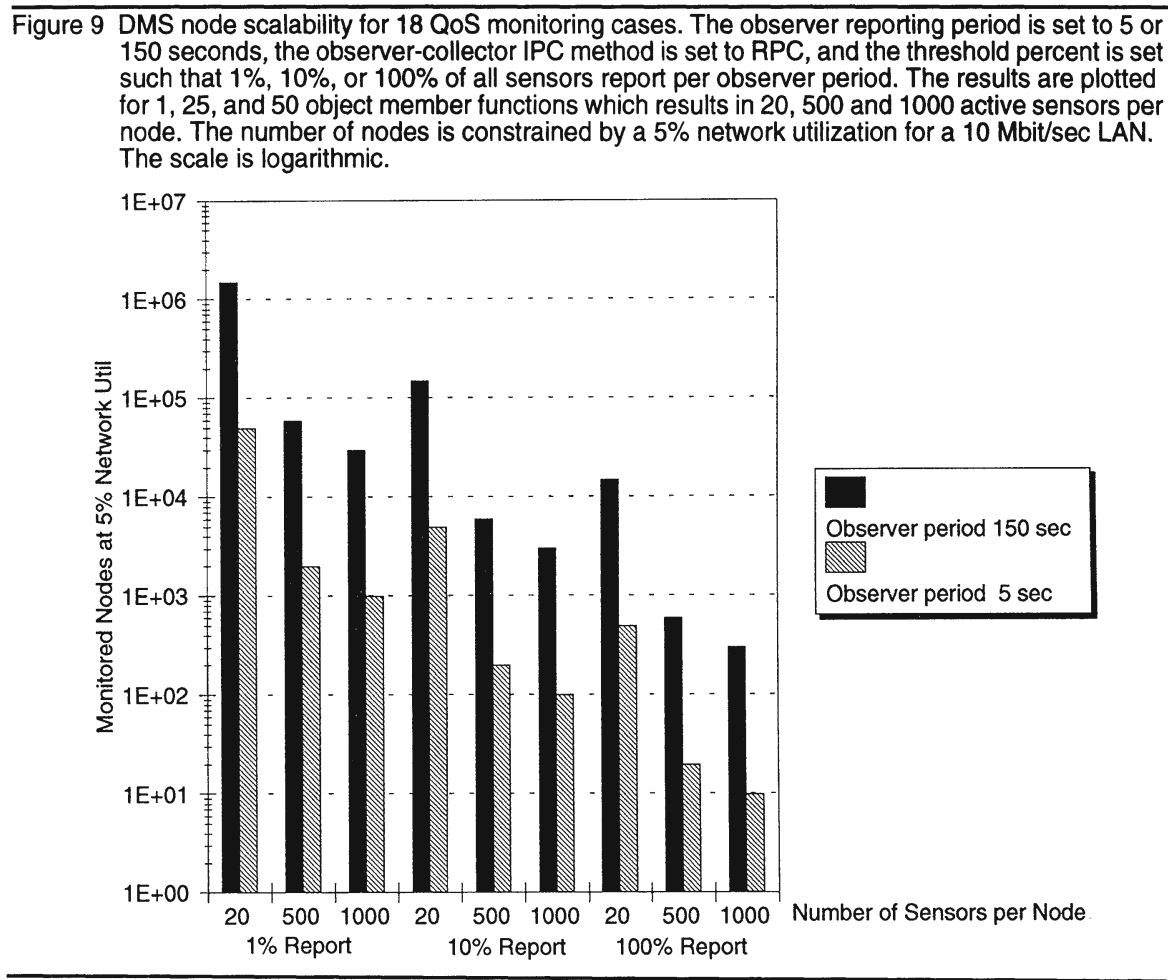

\section{SUMMARY AND CONCLUSIONS}

In this paper we present a predictive model for the overhead for the distributed application 
performance monitoring system DMS. DMS can be used to monitor the quality of service provided by distributed application systems, characterizing workloads, and building performance models. We identify several QoS and model building scenarios and explore their impact on system utilizations and workload responsiveness.

The processor utilization for DMS is dominated by its collector and observer costs. The overhead due to sensor instrumentation does not appear to be significant. This is because the collector and observers must communicate information and the communication costs are much greater than sensor costs. For our scenarios the processor utilization of DMS was less than $4.5 \%$ even for our most severe case. The impact of DMS on workload responsiveness is less than $5 \%$. We assumed the use of a 10 Mbit Ethernet LAN and concluded that network utilization by a single node was as low as $0.12 \%$. Our choice of reporting period was pessimistic; consequently, we believe that the impact of DMS will be less than the estimates described here.

The scalability of DMS was considered as a function of the number of member functions per node and the monitoring scenario. The number of nodes that can be supported without exceeding a 5\% network utilization is estimated and exceeds $10^{5}$ nodes for several scenarios. An advantage of DMS as opposed to polling based performance management mechanisms is that when performance degrades, the amount of information passed over a network remains bounded by the observation period. This helps avoid network storms at the times when performance has already degraded and reliable performance data is crucial.

From the results DMS appears well suited for QoS monitoring and for model building. Future work includes validating the models for DMS using measurement data from large environments.

\section{Acknowledgments}

We wish to acknowledge Joe Martinka, Steve Saunders, and Tracy Sienknecht of Hewlett-Packard for their contributions to the design and implementation of the DMS; and Jeff Taylor of Carleton University for helping to document and explore early versions of the model.

\section{REFERENCES}

1 ISO/IEC JTC1/SC21/WG7 N885, "Reference Model for Open Distributed Processing - Part 1: Overview and Guide to Use," November 1993.

2 P. Dauphin, R. Hofmann, R. Klar, B. Mohr, A. Quick, M. Siegle, and F. Sötz, ZM4/Simple: A General Approach to Performance Measurement and Evaluation of Distributed Systems, Readings in Distributed Computing Systems, IEEE Computer Society Press, Los Alamitos, CA, 1994, pp 286309.

3 G. Franks, A. Hubbard, S. Majumdar, D. Petriu, J. Rolia, and C.M. Woodside, "A Toolset for Performance Engineering and Software Design of Client-Server Systems", to appear in Performance Evaluation Journal, 1995.

4 R. Friedrich, "The Requirements for the Performance Instrumentation of the DCE RPC and CDS Services," OSF DCE RFC 32.0, June 1993.

5 R. Friedrich, S. Saunders, G. Zaidenweber, D. Bachmann, and S. Blumson, "Standardized Performance Instrumentation and Interface Specification for Monitoring DCE Based Applications," OSF DCE RFC 33.0, November 1994.

6 R. Friedrich, J. Martinka, T. Sienknecht, and S. Saunders, "Integration of Performance Measurement and Modeling for Open Distributed Processing," Open Distributed Processing, K. Raymond and L. Armstrong (editors), Chapman and Hall (London), pp. 347-358, 1995.

7 R. Jain, and I. Chlamtac, "The $\mathrm{P}^{2}$ Algorithm for Dynamic Calculation of Quantiles and Histograms without Storing Observations," Communications of the ACM, Vol. 28, No. 10, pp. 1076-1085.

8 F. Lange, R. Kroeger, and M. Gergeleit, JEWEL: Design and Implementation of a Distributed Measurement System, IEEE Transactions on Parallel and Distributed Systems, Volume 3 Number 6, November 1992, pp 657-671.

9 E. Lazowska, J. Zahoran, G. Graham, and K. Sevcik, Quantitative System Performance: Computer System Analysis Using Queueing Network Models, Prentice Hall, Inc., Englewood Cliffs, NJ, 1984.

$10 \mathrm{~J}$. Rolia, "Distributed Application Performance, Metrics and Management," Open Distributed Processing, II (C-20), J. de Meer, B. Mahr and S. Storp (Editors). Elsevier Science B.V. (NorthHolland), pp. 235-246, 1994.

11 J. Rolia and K. Sevcik, "The Method of Layers," IEEE Transactions on Software Engineering, Vol. 21, No. 8, August 1995, pp. 689-700.

12 B. Schroeder, “On-line Monitoring: A Tutorial," Computer, Vol. 28, No. 6, June 1995, pp.72-78. 